\section{Ultrawideband pulse generation using hybrid photonic microwave filters}

Hongwei Chen, Minghua Chen, and Shizhong Xie

Tsinghua University, Department of Electronic Engineering, Beijing, 100084, China

E-mail: chenhw@tsinghua.edu.cn

\begin{abstract}
A novel ultrawideband (UWB) pulse generation method using hybrid photonic microwave filters is proposed and demonstrated. Both electrical and optical delay are employed to construct microwave filters. The coefficients can be set to be either positive or negative by fiber chromatic dispersion and polarization modulation techniques, respectively. The generated UWB pulse has bandwidth larger than $1.8 \mathrm{GHz}$ and can be used in multiband modulation UWBover-fiber systems. () 2008 Society of Photo-Optical Instrumentation Engineers.
\end{abstract}

[DOI: 10.1117/1.2902452]

Subject terms: ultrawideband; photonic microwave filters; UWB over fiber.

Paper 070964LR received Dec. 6, 2007; revised manuscript received Jan. 11, 2008; accepted for publication Jan. 23, 2008; published online Apr. 30, 2008.

\section{Introduction}

Ultrawideband (UWB) technology is emerging as a solution for future wideband personal access networks (PANs). ${ }^{1,2}$ Its advantages over traditional wireless communication technologies include lower power consumption, higher bit rate, and immunity to multipath fading. ${ }^{3}$ In UWB systems, carrier-free impulse modulation is very attractive because it not only avoids complicated frequency mixer, intermediate-frequency carrier, and filter circuits, but also has better passthrough characteristics due to its baseband transmission. As one of the radio-over-fiber techniques, UWB over fiber can be a candidate solution for future wideband access networks. ${ }^{4}$

Many works focused on optical generation and distribution of monocycle and doublet pulses have been reported. ${ }^{5-10}$ These schemes can only be used in singleband UWB systems. In fact, an alternative signaling format for single-band UWB impulse radio is to use multiband (MB) UWB waveforms, which were proposed for wireless personal area networks (WPANs) under IEEE 802.15. Multiband UWB is one of the possible techniques where the entire UWB single band is split into several nonoverlapping smaller subbands, each of which satisfies the Federal Communications Commission (FCC) bandwidth requirement. With the entire bandwidth divided into several nonoverlapping subbands, multiband UWB systems benefit from flexibility, avoiding interference and multiple accesses.

The multiband UWB signal design is dependent on digital filters with lots of coefficients. ${ }^{2}$ Recently, great interest in photonic microwave filtering technique has arisen, which can process high-frequency microwave signals in the opti-

0091-3286/2008/\$25.00 @ 2008 SPIE cal domain. ${ }^{11-13}$ However, to the best of our knowledge, there has been no attempt to generate multiband UWB pulses by using photonic microwave filters.

In this letter, a novel UWB pulse generation technique using hybrid photonic microwave filters is proposed and demonstrated. Both electrical and optical delay are employed to construct microwave filters. The coefficients can be set to be either positive or negative by fiber chromatic dispersion and polarization modulation techniques, respectively, with totally incoherent operation. The bandwidth of the generated UWB pulse is larger than $1.8 \mathrm{GHz}$, and it can be used in multiband modulation UWB systems.

\section{Principle and Experiment}

The experimental setup of our proposed scheme is shown in Fig. 1 with waveforms at different locations. Continuous waves from three laser diodes (LDs), working at 1539.77, 1545.32, and $1550.92 \mathrm{~nm}$, respectively, are multiplexed after passing through polarization controllers and variable optical attenuators to keep identical polarization and power. The polarization is tuned to be linear and launched at 45 deg relative to the principal axis of the $\mathrm{LiNbO}_{3}$ phase modulator (PM), so that the optical waves can be polarization-modulated. The driving signal for the PM comes from a pulse generator with a fixed pattern: one 1 every 128 bits with bit rate of $8.3 \mathrm{GHz}$. After a delay unit, the pattern becomes one 101010 sequence every 128 bits, which is equivalent to a transversal filter with coefficient

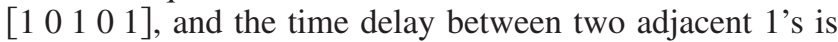
about $240 \mathrm{ps}$ (two-bit length). The $z$-transform system function is $H_{1}(z)=1+z^{-2}+z^{-4}$. If the driving voltage is set to $V_{\pi}$, the signal polarization will rotate to the orthogonal direction at the output of the PM. So at the input orientation there are negative pulses with bit 1 , while at the orthogonal orientation there are positive pulses [1 0$]$. The output pulse

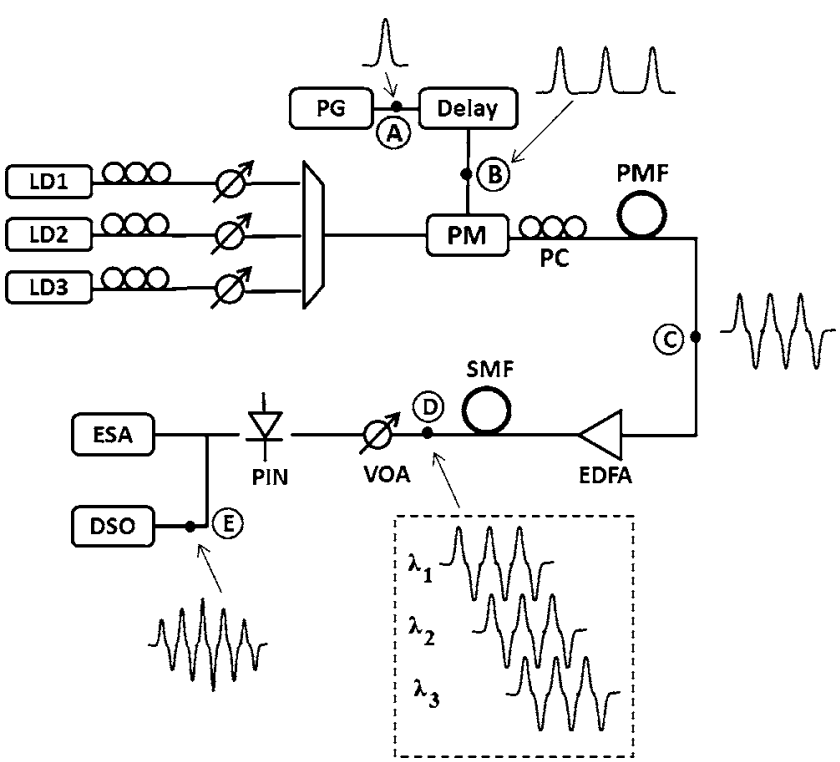

Fig. 1 Experimental setup. DSO, digital sampling oscillator; EDFA, erbium-doped fiber amplifier; ESA, electrical spectrum analyzer; LD, laser diode; PC, polarization controller; PG, pulse generator; PM, phase modulator; PMF, polarization-maintaining fiber; SMF, singlemode fiber; VOA, variable optical attenuator. Polarization difference is not shown for simplicity. 


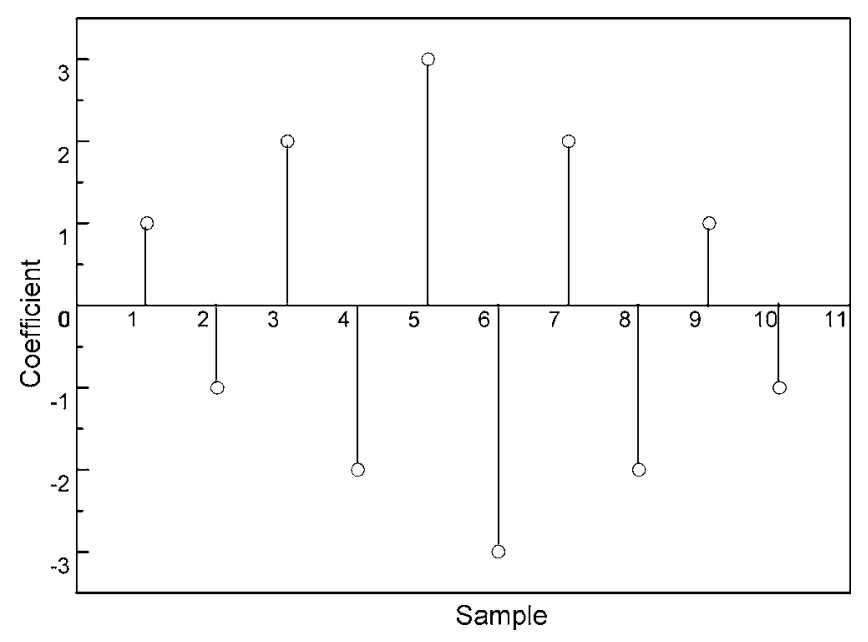

Fig. 2 Coefficients of hybrid photonic microwave filter.

orientation of the phase modulator is adjusted by a PC and fed into a section of polarization-maintaining fiber (PMF) with length $90 \mathrm{~m}$. The orthogonal pulses are made to align with the principal axes of the PMF so they can be delayed properly. The beat length of the PMF is $3.8 \mathrm{~mm}$, and the time delay between the two principal states of polarization (PSPs) is about $120 \mathrm{ps}$. Thus, the PMF acts as the second transversal filter with negative coefficient of $[1-1]$. The $z$-transform system function is $H_{2}(z)=1-z^{-1}$. The signal is then boosted by an erbium-doped fiber amplifier and fed into a $2.5-\mathrm{km}$ single-mode fiber (SMF). The wavelengthdependent time delay due to fiber chromatic dispersion is $T=D \times L \times \Delta \lambda$, where $D$ is the dispersion parameter [SMF: $17 \mathrm{ps} /(\mathrm{nm} \mathrm{km})], L$ is the fiber length $(2.5 \mathrm{~km})$, and $\Delta \lambda$ is the wavelength spacing between optical sources $(5.6 \mathrm{~nm})$. So the time delay in the SMF is about $240 \mathrm{ps}$, which is twice that in PMF. Because there are three optical wavelengths, the filter coefficient is $\left[\begin{array}{lllll}1 & 0 & 1 & 0 & 1\end{array}\right]$ and the $z$-transform system function is $H_{3}(z)=1+z^{-2}+z^{-4}$. Thus, the signal after electrical time delay, polarization time delay, and chromatic-dispersion-induced time delay is equivalent to one that has passed three transversal filters with a system function of

$$
\begin{aligned}
H(z)= & H_{1}(z) \times H_{2}(z) \times H_{3}(z) \\
= & 1-z^{-1}+2 z^{-2}-2 z^{-3}+3 z^{-4}-3 z^{-5}+2 z^{-6}-2 z^{-7}+z^{-8} \\
& -z^{-9}
\end{aligned}
$$

and is shown in Fig. 2. It can be seen that there is a triangular apodization profile of the coefficients, which is helpful in filter sidelobe suppression. The calculated spectrum of such a filter is shown in Fig. 3. The sidelobe is suppressed by more than $20 \mathrm{~dB}$, and the two main lobes are kept in the range of 0 to $10 \mathrm{GHz}$. Because the pulse pattern is triggered at $8.33-\mathrm{GHz}$ repetition frequency, the main part of spectrum is located in the $0-$ to $7-\mathrm{GHz}$ range. One of the main lobes can be used for spectrum forming, and the other is of no use.

The optical signal after the SMF is attenuated by a VOA and detected by a pin detector with bandwidth of $30 \mathrm{GHz}$. A digital sampling oscilloscope (DSO) and an electrical

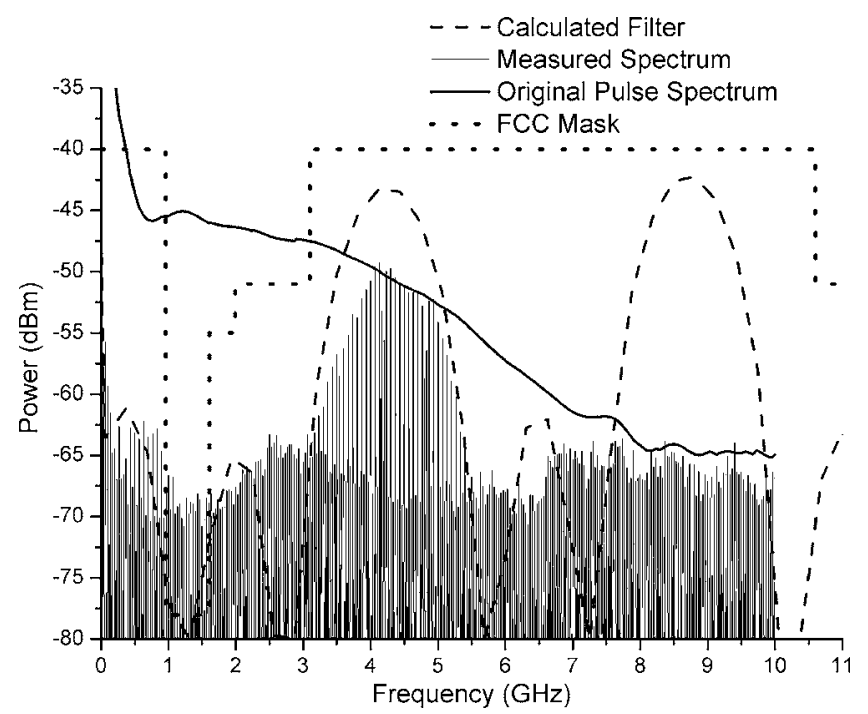

Fig. 3 Spectra of calculated filter, measured signal, original pulse, and FCC mask.

spectrum analyzer (ESA) are used to measure the time profile and spectrum of the detected signal. Figure 3 also shows the measured spectrum of the generated pulse, which fits the filter profile quite well. The fractional bandwidth is determined as

$B_{f}=2 \frac{f_{H}-f_{L}}{f_{H}+f_{L}}$,

where $f_{L}$ and $f_{H}$ are the lower and higher -10 -dB points in the spectrum (3.36 and $5.2 \mathrm{GHz}$ ), respectively. So the $-10-\mathrm{dB}$ bandwidth is $1.84 \mathrm{GHz}$, and the fractional bandwidth of the generated pulse is $43 \%$. This spectrum falls within the frequency mask for UWB defined by the FCC and can be used for multiband UWB systems. However, from Fig. 3, the signal-to-noise ratio (SNR) of the generated pulse is still low, due mainly to the low saturation power and large bandwidth of the optical detector. The saturation power of our optical detector is about $3 \mathrm{dBm}$, and the bandwidth is $40 \mathrm{GHz}$. We tuned the optical power

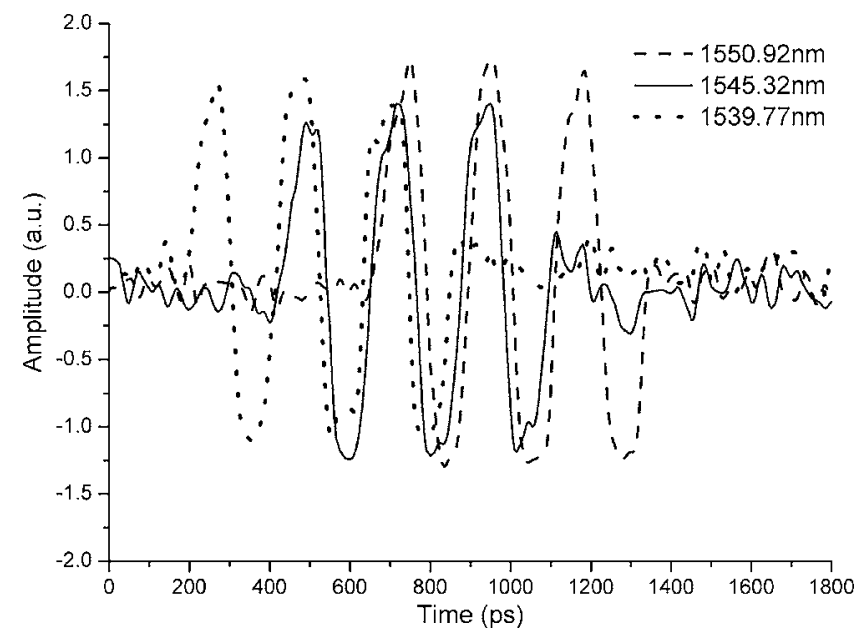

Fig. 4 Time profiles of three wavelengths. 


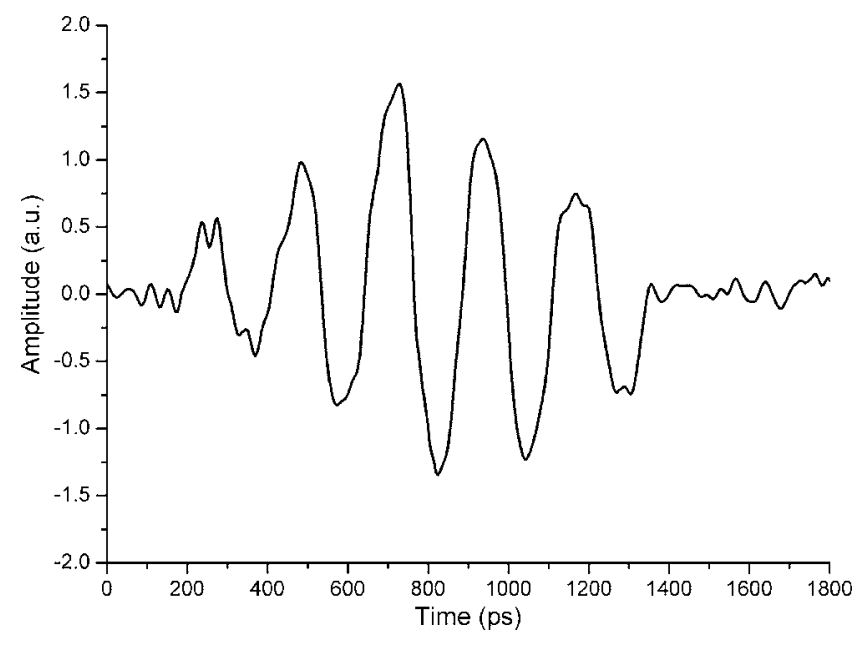

Fig. 5 Time profile of generated UWB pulse.

to $0 \mathrm{dBm}$ for UWB pulse generation. With a high-quality optical-to-electrical detector, the SNR can be markedly improved.

Figure 4 shows the time profiles of different wavelengths. The signal of each wavelength is modulated with six bits $(1,-1,1,-1,1,-1)$. After the SMF, signals at adjacent wavelengths are delayed by one bit so that they can be combined correctly as shown in Fig. 1. The final detected pulse is shown in Fig. 5. There is also apodization on the pattern amplitude, and the duration of this pulse is about 1200 ps.

\section{Conclusion}

A novel method for optical multiband UWB pulse generation has been proposed and demonstrated, which utilizes both electrical and optical delay to construct microwave filters. A ten-tap transversal filter is realized with only three optical sources. The generated UWB pulse is the superposition of pulse trains from three wavelengths. From the frequency-domain point of view, the superposition can be seen as apodization of transversal filter coefficients, so that the sidelobe can be significantly suppressed. Consequently, the pulse spectrum is in line with the FCC mask. Different bands of UWB pulses can be generated by changing the modulation frequency and time delay properly. The obtained pulse achieved a bandwidth of $1.8 \mathrm{GHz}$ and a fractional bandwidth of $43 \%$, which can be used in multiband UWB-over-fiber systems.

\section{Acknowledgement}

This work is supported by the Tsinghua Basic Research Fund under grant JC2007020, the NSFC under contract 60736002, the National 863 Program of China under Contract 2007AA01Z264, and the Open Fund of the Key Laboratory of Optical Communication and Lightwave Technologies (Beijing University of Posts and Telecommunications), Ministry of Education, China.

\section{References}

1. G. R. Aiello and G. D. Rogerson, "Ultra-wideband wireless systems," IEEE Microw. Mag. 4, 36-47 (Jun. 2003).

2. H. Arslan, Z. N. Chen, and M.-G. Di Benedetto, "Ultra Wideband Wireless Communication," John Wiley \& Sons, Inc., Hoboken, NJ (2006).

3. M. Z. Win and R. A. Scholtz, "Ultra-wide bandwidth time hopping spread spectrum impulse radio for wireless multiple-access communications," IEEE Trans. Commun. 48, 679-689 (Apr. 2000).

4. D. Porcino and W. Hirt, "Ultra-wideband radio technology: potential and challenges ahead," IEEE Commun. Mag. 41, 66-74 (Jul. 2003).

5. I. S. Lin, J. D. McKinney, and A. M. Weiner, "Photonic synthesis of broadband microwave arbitrary waveforms applicable to ultrawideband communication," IEEE Microw. Wirel. Compon. Lett. 15 226-228 (Apr. 2005).

6. W. P. Lin and Y. C. Chen, "Design of a new optical impulse radio system for ultra-wideband wireless communications," IEEE J. Sel. Top. Quantum Electron. 12, 882-887 (Jul./Aug. 2006).

7. T. Kawanishi, T. Sakamoto, and M. Izutsu, "Ultra-wide-band radio signal generation using optical frequency-shift-keying technique," IEEE Microw. Wirel. Compon. Lett. 15, 153-155 (Mar. 2005).

8. F. Zeng, Q. Wang, and J. Yao, "An approach to all-optical UWB pulse generation," in IEEE Int. Top. Mtg. on Microwave Photonics, $M W P^{\prime} 06$, p. 13.

9. Q. Wang, F. Zeng, S. Blais, and J. Yao, "Optical ultrawideband monocycle pulse generation based on cross-gain modulation in a semiconductor optical amplifier," Opt. Lett. 31, 3083-3085 (Nov. 2006).

10. H. Chen, M. Chen, C. Qiu, J. Zhang, and S. Xie, "UWB monocycle pulse generation by optical polarisation time delay method," Electron. Lett. 43(9), 542-543 (2007).

11. J. Capmany, B. Ortega, and D. Pastor, "A tutorial on microwave photonic filters," J. Lightwave Technol. 24(1), 201-229 (2006).

12. J. Mora, M. V. Andres, J. L. Cruz, B. Ortega, J. Capmany, D. Pastor, and S. Sales, "Tunable all-optical negative multitap microwave filters based on uniform fiber Bragg gratings," Opt. Lett. 28, 1308-1310 (Aug. 2003).

13. C. K. Oh, T.-Y. Kim, S. H. Baek, and C.-S. Park, "Photonic microwave notch filter using cross polarization modulation in highly nonlinear fiber and polarization-dependent optical delay in high birefringence fiber," Opt. Express 14(15), 6628-6633 (2006). 